\title{
AVALIAÇÃO DA CONSISTÊNCIA DA INDEXAÇÃO EM BIBLIOTECAS UNIVERSITÁRIAS FEDERAIS DA REGIÃO NORDESTE DO BRASIL
}

\author{
Roberta Cristina Dal'Evedove Tartarotti* \\ Universidade Estadual Paulista - UNESP - Brasil. \\ Paula Regina Dal'Evedove** \\ Universidade Federal de São Carlos - UFSCar - Brasil. \\ Mariângela Spotti Lopes Fujita ${ }^{* * *}$ \\ Universidade Estadual Paulista - UNESP - Brasil.
}

\begin{abstract}
Resumo: Nos sistemas de recuperação da informação automatizados, o uso adequado da linguagem documental é fundamental, pois possibilita a representação de conteúdos documentais compatíveis com as solicitações de pesquisas dos usuários em uma determinada área científica especializada. Por meio da metodologia quantitativa de Avaliação da Indexação, foi possível realizar um estudo diagnóstico da representação temática de livros em catálogos coletivos online de bibliotecas universitárias. O universo de pesquisa constituiu-se de 9 bibliotecas universitárias federais da Região Nordeste do Brasil. Os resultados apontam uma média de 46,99\% de índice de consistência para o índice relaxado, enquanto que a média foi de $39,21 \%$ com o índice rígido. Como considerações finais, ainda são diversos os fatores que influenciam na qualidade da indexação, sendo necessários mais estudos teórico-metodológicos na área. Além disso, outros estudos complementares de outras regiões brasileiras possibilitariam um panorama atual e mais amplo da qualidade do processo de indexação em sistemas de recuperação da informação de bibliotecas universitárias no contexto brasileiro.
\end{abstract}

Palavras-chave: Avaliação da indexação; consistência na indexação; catálogos coletivos online; bibliotecas universitárias brasileiras.

Título: EVALUACIÓN DE LA CONSISTENCIA EN LA INDIZACIÓN EN BIBLIOTECAS UNIVERSITARIAS FEDERALES DE LA REGIÓN NORDESTE DE BRASIL.

Resumen: En los sistemas de recuperación automatizados, el uso adecuado del lenguaje de indización es esencial, ya que permite la representación de contenido de documentos compatible con las solicitudes de búsqueda de los usuarios en un área de especialización. A través de la metodologia cuantitativa de la Evaluación de la Indización, fue posible llevar a cabo un estudio diagnóstico de la representación temática de libros en catálogos colectivos en línea de bibliotecas universitarias. El universo de estudio fueron 9 bibliotecas universitarias federales en la Región Nordeste de Brasil. Los resultados muestran un promedio de 46,99\% de consistencia entre los índices para el índice relajado, mientras que el promedio fue de $39,21 \%$ con el índice rígido. Como consideraciones finales, aún son diversos los factores que influyen en la calidad de la indización, siendo necesarios más estúdios teóricosmetodológicos en el área. Además, otros estudios complementarios de otras regiones brasileñas posibilitarian un panorama actual y más amplio de la calidad del proceso de indización en sistemas de recuperación de información de las bibliotecas universitarias en el contexto brasileño.

Palabras clave: Evaluación de la indización; consistencia en la indización; catálogos colectivos en línea; bibliotecas universitarias brasileñas.

Title: EVALUATION OF INDEXING CONSISTENCY AT FEDERAL UNIVERSITY LIBRARIES IN NORTHWESTERN OF BRAZIL.

Abstract: In automated retrieval systems, the proper use of the indexing language is essential because it allows the representation of document content compatible with the users' search requests in an area of specialization. Through the quantitative methodology of the Evaluation of Indexing, it was possible to carry out a diagnostic study of thematic representation of books in online catalogs of university libraries. The universe of study was 9 federal university libraries in the Northeast Region of Brazil. The results showed an average of $46.99 \%$ consistency between the indices for the relaxed index, while the average was $39.21 \%$ with the rigid index. As final considerations, there are still several factors that influence the quality of the indexing, being necessary more theoretical-methodological studies in the area. In addition, other complementary studies from other Brazilian regions could provide a current and broader picture of the quality of the indexing process in information retrieval systems of university libraries in the Brazilian context.

Keywords: Indexing evaluation; indexing consistency; online catalogs; brazilian university libraries.

\footnotetext{
* roberta_tartarotti@yahoo.com.br

** .dallevedove@gmail.com

**** fujita@marilia.unesp.br
}

Recibido: 11-03-2016; 2 $2^{\mathrm{a}}$ versión: 09-01-2017; aceptado: 07-02-2017.

DAL'EVEDOVE TARTAROTTI, R.C.; DAL'EVEDOVE, P.R. y FUJITA, M.S.L. Avaliação da consistência da indexação em bibliotecas universitárias federais da Região Nordeste do Brasil. Anales de Documentación, 2017, vol. 20, $\mathrm{n}^{\circ}$ 1. Disponible en: http://dx.doi.org/10.6018/analesdoc.20.1.253201. 


\section{INTRODUÇÃ̃O}

A indexação de assunto é considerada um dos aspectos mais importantes de um sistema de recuperação de informação. Entretanto, a adequação e qualidade deste processo são de difíceis medições e avaliações, considerando-se que não existe uma única forma de indexar devido à existência de diversas variáveis, como o próprio indexador, o tipo de documento, as características e particularidades do contexto e a política de indexação da instituição.

O método para avaliar a amplitude destas variações consiste em obter a medida da consistência ou concordância existente na realização da indexação documental. Quando as comparações são realizadas entre o resultado de um indexador na análise de um mesmo documento em períodos diferentes, é conhecido como consistência intraindexador; enquanto que a confrontação entre o resultado de vários indexadores na análise de um mesmo documento se denomina consistência interindexador (Abad García; Abad Pérez; Benavent, 1998; Gil Leiva, 2008).

A indexação pode ser definida como um processo formado por subprocessos ou etapas que tem como objetivo identificar o conteúdo de um documento, por meio de uma metalinguagem construída - a linguagem de indexação com o intuito de promover a efetiva recuperação da informação. Em termos teóricos, qualquer recurso informacional é passível de ser indexado ou representado por conceitos. Esta ação viabiliza que a informação documental seja acessada e recuperada para usos distintos por sujeitos com interesses específicos.

No contexto de bibliotecas universitárias, a eficiente recuperação da informação em catálogos coletivos online está vinculada ao processo de indexação, em que a qualidade da representação de assunto está diretamente relacionada à qualidade da análise temática, considerando sua complexidade inerente ao fazer profissional. Os problemas apontados por Lancaster (2004) ainda são presentes na indexação, muito em detrimento das ações empregadas pelos indexadores frente ao documento, sendo estas relativas à interpretação errônea ou omissão do assunto do documento na análise conceitual, escolha de termos inadequados para a tradução do conteúdo do documento, falta de especificidade no vocabulário e falta de especificidade na indexação.

Ao considerarmos que a solução de muitas das problemáticas que cercam a representação de assunto perpassa pelo avanço de pesquisas acerca da consistência da indexação, entendendo-se "qualidade da indexação" em termos de eficácia da recuperação (Cooper, 1969; Leonard, 1977), torna-se relevante o encaminhamento de pesquisas destinadas à temática, tendo a avaliação da consistência como sinônimo de confiabilidade do processo. Embora alguns estudos na área indiquem a dificuldade em medir a qualidade da indexação, muito em razão da singularidade do processo (Funk; Reid; McGoogan, 1983), outros sinalizam que alcançar a consistência é contribuir para uma recuperação eficiente (Saarti, 2002).

Em vista disso, objetiva-se nesta investigação apresentar os resultados advindos com a avaliação da consistência da indexação de livros em catálogos coletivos online de bibliotecas universitárias federais da Região Nordeste do Brasil por meio da metodologia quantitativa da Avaliação da Indexação (AI), pela qual é possível medir e avaliar os aspectos intrínsecos e extrínsecos do processo de indexação para fins de aprimoramento da atividade no contexto acadêmico.

\section{A METODOLOGIA QUANTITATIVA DA AVALIAÇÃO DA INDEXAÇÃO}

No âmbito do tratamento temático da informação documental, destaca-se a relevância da avaliação do processo de indexação como mecanismo de análise da qualidade dos produtos e serviços gerados no contexto de bibliotecas universitárias. Por meio da metodologia quantitativa da AI, é possível obter um feedback da representação da informação com o intuito de aprimorá-la de acordo com a demanda do sistema, contribuindo para a aplicabilidade e sistematização da atividade de indexação em bibliotecas universitárias.

Segundo Wolfram, Olson e Bloom (2009), faz parte da cultura tradicional da Ciência da Informação que o processo de indexação permita representar com exatidão o conteúdo de um documento. Idealmente, se dois indexadores analisam o mesmo documento, os descritores serão os mesmos para ambas as indexações, ocorrendo um alto nível de concordância ou consistência.

Entretanto, os autores salientam que a indexação não é tão simples assim. Décadas de pesquisa sobre a consistência entre indexadores, bem como em torno do mesmo indexador em diferentes momentos permitiram o desenvolvimento de uma série de medidas para avaliar a consistência entre indexadores. Além disso, os estudos com indexadores humanos têm sido comparados não apenas entre os próprios indexadores, mas também com o resultado dos sistemas de indexação automática. De forma geral, embora os resultados destas pesquisas apresentaram elevados índices de 
inconsistência, de acordo com Abad García, Abad Pérez e Benavent (1998) a metodologia da AI permite analisar a atividade de indexação em um sistema de recuperação da informação, sendo relevante tanto para os produtores da informação (conhecer aspectos reais da realização de determinadas atividades e propor meios para sua melhora), como para seus usuários (conhecer como funciona o sistema de informação e assim poder utilizá-lo do melhor modo possível).

Segundo Olson e Wolfram (2008), as primeiras pesquisas em torno da AI surgiram por volta de 1950, década de maior publicação, culminando no estudo de Zunde e Dexter (1969). No geral, estas investigações possuem cunho teórico, em busca de respostas sobre as diferenças entre os resultados obtidos na indexação ou cunho prático, direcionadas à quantificação da consistência entre duas ou mais indexações por meio de equações:

A outra parte importante da literatura está dedicada à quantificação da consistência, ou seja, à obtenção de índices de consistência que vão desde o 1 ao 100\%, mediante fórmulas diversas de semelhança entre indexações, apoiando-se em diferentes aspectos como a experiência (indexadores principiantes ou indexadores experientes) ou as tipologias documentais (livros, patentes, artigos de periódicos, fotografias, etc.) (Gil Leiva; Rubi; Fujita, 2008, p. 235).

Define-se a consistência como o "grau de semelhança na representação da informação documental de um documento por meio de termos de indexação selecionados por um ou vários indexadores, resultando em um índice de consistência" (Gil Leiva; Rubi; Fujita, 2008, p. 234) ou "uma medida quantitativa do grau em que dois ou mais indexadores identificam os conceitos importantes contidos em um documento e representam estes conceitos usando códigos/termos idênticos" (Leonard, 1977, p. 2).

De acordo com a literatura especializada, os resultados do processo de indexação em bibliotecas universitárias podem ser obtidos por meio de diferentes abordagens, expostos a seguir.

\subsection{Métodos de Avaliação da Indexação}

A metodologia da AI comporta dois aspectos principais: intrínseco e extrínseco, em que cada um comporta subcategorias de análise, sintetizadas por Gil Leiva (2008).

A Avaliação Intrínseca está relacionada com o resultado da indexação, com a finalidade de conhecer a sua qualidade:

[...] um conjunto de tarefas centradas no resultado da indexação (descritores, cabeçalhos, subcabeçalhos ou identificadores), com a finalidade de conhecer sua qualidade. A avaliação intrínseca de indexação pode ser qualitativa, isto é, por meio de valorização e consenso entre [profissionais] experientes, ou quantitativa, através de fórmulas (Gil Leiva, 2008).

A Avaliação Intrínseca subdivide-se em dois tipos:

- Avaliação Intrínseca Qualitativa: caracteriza-se por analisar os componentes inerentes que proporcionam a qualidade da indexação, como:

- Exaustividade: quando se extrai todos os conceitos caracterizadores do conteúdo integral dos documentos;

- Especificidade: que exista uma relação exata entre as unidades conceituais escolhidas e o(s) termo(s) escolhidos para representá-las.

- Correção: que não aconteçam erros de inclusão (um termo que não procede) e nem erros de omissão (a exclusão de um termo relevante).

- Perspectiva do usuário: que se considere os interesses e perspectivas dos usuários potenciais, para o qual, tem que responder à pergunta se os termos ou assunto designado, em seu conjunto ou separado, são legítimos para representar o conteúdo do documento.

- Avaliação Intrínseca Quantitativa: refere-se a uma reindexação de um conjunto de documentos repetindo-se, na medida do possível, as condições em que foi produzida a primeira indexação, para conseguir índices de consistência entre duas indexações por meio de métodos ou fórmulas matemáticas. Para Inácio (2012), este tipo de avaliação possibilita a análise dos índices de consistência entre indexadores, bem como sobre política de indexação, linguagem de indexação, condições de trabalho e usuários potenciais. Este tipo de avaliação subdividese em dois tipos: 
- Avaliação Intrínseca Quantitativa Mediante a Interconsistência: compara a indexação de um mesmo documento realizada por dois ou mais profissionais (consistência interindexador).

- Avaliação Intrínseca Quantitativa Mediante a Intraconsistência: compara a indexação de um mesmo documento realizada pelo mesmo profissional em diferentes momentos no tempo (consistência intraindexador).

Em síntese, enquanto a Avaliação Intrínseca Qualitativa baseia-se em uma investigação da atuação profissional dos indexadores, a Avaliação Intrínseca Quantitativa caracteriza-se pela aplicação de fórmulas matemáticas.

A consistência na indexação pode ser estudada como referência a um único indexador ou a vários. Quando um profissional indexa um mesmo documento, em diferentes momentos temporais, falamos de intraconsistência ou consistência intraindexador. Em segundo lugar, quando vários profissionais indexam um mesmo documento visando comparar seu resultado ou quando se enfrentam duas indexações de diferentes indexadores, falamos de interconsistência ou consistência interindexador (Gil Leiva; Rubi; Fujita, 2008, p. 234).

Em contrapartida, segundo Gil Leiva (2008) a Avaliação Extrínseca mede o resultado da indexação para comparálo com a indexação de outras bibliotecas que igualmente indexaram o mesmo documento (interconsistência) ou mediante a recuperação (exaustividade e precisão). A Avaliação Extrínseca subdivide-se em dois tipos:

- Avaliação Extrínseca Mediante a Interconsistência: compara a indexação de um mesmo documento realizada por duas ou mais bibliotecas.

- Avaliação Extrínseca Mediante a Recuperação: compara duas indexações procedentes de um mesmo sistema de recuperação da informação ou dois sistemas diferentes.

Na Avaliação Extrínseca Mediante a Recuperação, a comparação pode ser feita tanto entre uma indexação manual e uma automática como entre duas automáticas ou duas manuais, ou seja, comparar duas bases de dados que tenham conteúdo idêntico, menos os campos que se referem à indexação, apresentando resultados referentes à exaustividade e à precisão na recuperação com a da seguinte equação (Gil Leiva, 2008):

Exaustividade $=$ número de documentos relevantes recuperados número total de documentos relevantes na coleção

Precisão $=\frac{\text { número de documentos relevantes recuperados }}{\text { número total de documentos recuperados }}$

Diante destes métodos, com o intuito de realizar a AI documental em catálogos coletivos online de bibliotecas universitárias, optou-se pela Avaliação Intrínseca Quantitativa pela Interconsistência ou Avaliação Interbibliotecário.

A consistência interindexador "é uma medida quantitativa do grau de concordância entre diferentes indexadores indexando o mesmo documento dentro de um sistema de recuperação de informação" (Hughes, 2011, p. 9). Ainda para o autor, a literatura aponta uma correlação experimental entre a consistência da indexação e a eficácia de recuperação dos documentos e considera a consistência como um marco de qualidade na prestação de serviços aos usuários.

Em geral, a avaliação intrínseca quantitativa visa conhecer o grau de semelhança entre os indexadores. Gil Leiva, Rubi e Fujita (2008, p. 236) indicam que o grau de consistência será maior, quanto mais semelhantes forem as indexações e esclarecem que, se comparadas, "as categorias oscilam de 0 a 1 ou de 0 a 100 , quando se traslada a um $\%$ ".

A consistência interindexador tem se revelado útil para avaliar o desempenho da indexação entre indexadores, sendo formulada de maneira diferente por alguns autores (Choi, 2011). A aplicação de fórmulas de avaliação quantitativas de interconsistência proporcionam medidas objetivas, por meio das quais o nível de consistência entre dois (ou mais) indexadores pode ser determinado. Para Leininger (2000), talvez a maior fonte de variabilidade nos resultados dos estudos sobre a avaliação da interindexação é que nenhum cálculo matemático simples da consistência interindexador foi adotado como padrão. Por exemplo, Leonard (1977) discutiu sete deles e Rolling (1981) mais quatro. Os cálculos mais conhecidos são as de Hooper (1965) e Rolling (1981), que apresentam simples fórmulas dos termos atribuídos por cada indexador e os termos comuns entre as duas indexações. 


\section{PROCEDIMENTOS METODOLÓGICOS}

Nesta investigação, como parâmetro metodológico, foi utilizada a fórmula da consistência adaptada de Hooper (1965) por Gil Leiva (2008), permitindo uma comparação dos índices de consistência gerados (Quadro I). Na fórmula em destaque, o índice de consistência entre as duas indexações é obtido dividindo-se o número de termos comuns nas duas indexações pelo número de termos usados na indexação $\mathrm{A}$, somado ao número de termos usados na indexação $\mathrm{B}$ e subtraído novamente do número de termos comuns nas duas indexações.

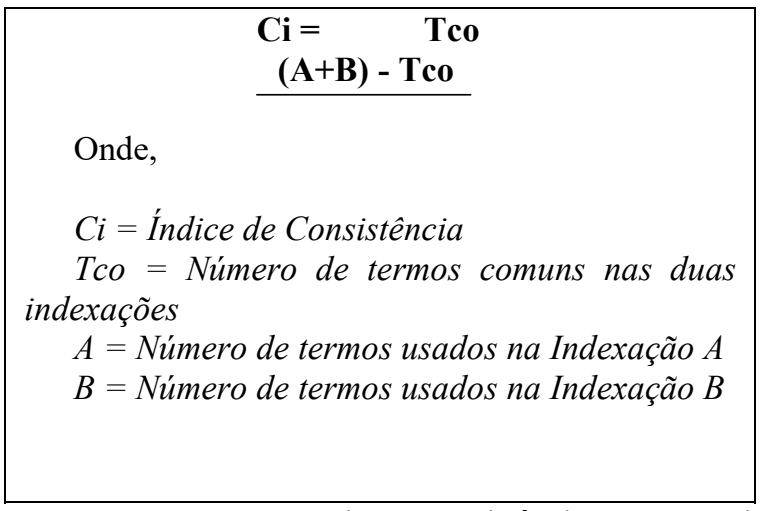

Quadro I. Fórmula para obter o índice de consistência entre duas indexações.

Para a comparação de uma amostra da representação temática nos catálogos coletivos online de bibliotecas universitárias brasileiras, foram selecionadas nove universidades da Região Nordeste do país (Quadro II). Realizaramse buscas por assunto nos catálogos coletivos online disponibilizados pelas respectivas universidades, visto que não se tem um mecanismo de busca de âmbito nacional que agregue todos os catálogos das instituições públicas de ensino superior do país. Assim, as buscas dos assuntos dos livros de várias áreas do conhecimento foram realizadas nos próprios catálogos coletivos online.

\begin{tabular}{|l|l|l|}
\hline Estado & Universidade & Sigla \\
\hline Alagoas & Universidade Federal de Alagoas & UFAL \\
Bahia & Universidade Federal da Bahia & UFBA \\
Ceará & Universidade Federal do Ceará & UFC \\
Maranhão & Universidade Federal do Maranhão & UFMA \\
Paraíba & Universidade Federal da Paraíba & UFPB \\
Pernambuco & Universidade Federal de Pernambuco & UFPE \\
Piauí & Universidade Federal do Piauí & UFPI \\
Rio Grande do Norte & Universidade Federal do Rio Grande do Norte & UFRN \\
Sergipe & Universidade Federal de Sergipe & UFS \\
\hline
\end{tabular}

Foram selecionados 10 títulos de livros, todos recuperados nos nove catálogos coletivos online, o que viabilizou a comparação tanto dos assuntos no campo 650 do registro MARC (o qual diz respeito aos assuntos controlados), como do número de chamada atribuídos pelas bibliotecas para cada documento em análise. Isto porque, no contexto de bibliotecas universitárias, o formato descritivo adotado é o catalográfico, grande parte em MARC21, no qual são introduzidos os resultados das operações de tratamento de forma e de conteúdo (Rubi, 2008).

Os cálculos dos índices de interconsistência (Gil Leiva; Rubi; Fujita, 2008) pautaram-se em dois tipos:

- Comparação flexível ou comparação relaxada: quando um assunto coincide com o assunto de outro documento, considera-se coincidência total (1); quando ocorre somente o cabeçalho ou subcabeçalho, considera-se a metade $(0,5)$ e quando não há nenhuma coincidência, o valor é 0 .

- Comparação rígida: quando o assunto determinado coincide completamente. 


\section{RESULTADOS E DISCUSSÃO}

Para a visualização comparativa da descrição temática, para cada título de livro foram elaborados Ensaios (Apêndices). Cada ensaio é composto de um quadro comparativo dos assuntos levantados e de uma tabela. Por sua vez, cada quadro dos ensaios é composto por 3 colunas e 10 linhas. Na primeira coluna, estão dispostas as siglas das bibliotecas universitárias pesquisadas. Na segunda coluna estão representados os assuntos recuperados nos registros. Em complementação, na terceira coluna está a classificação atribuída para o respectivo livro.

Concluída esta fase, aplicou-se a fórmula de Hopper (1965) adaptada por Gil Leiva (2008). Como produtos da AI, foram obtidos ensaios de interconsistência ou índices de consistência. Desse modo, cada tabela do ensaio apresenta três colunas. Na primeira coluna, foram dispostos os pares de bibliotecas. Na segunda coluna, apresenta-se o índice de consistência relaxado, ficando na terceira coluna o índice de consistência rígido.

Os resultados permitiram a comparação dos assuntos identificados durante o processo de representação temática de livros no contexto específico de bibliotecas universitárias, verificando inconsistências (discrepâncias) entre as indexações. Os índices de consistência acumulados dos 10 ensaios (relaxado e rígido) são apresentados abaixo, em ordem decrescente, conforme segue (Tabela I e II):

\begin{tabular}{l|l}
\hline Ensaios & $\begin{array}{l}\text { Índice de consistência } \\
\text { (relaxado) }\end{array}$ \\
\hline Ensaio 4 & 68,08 \\
\hline Ensaio 10 & 62,25 \\
\hline Ensaio 3 & 57,80 \\
\hline Ensaio 2 & 55,11 \\
\hline Ensaio 1 & 48,33 \\
\hline Ensaio 5 & 44,38 \\
\hline Ensaio 9 & 41,14 \\
\hline Ensaio 6 & 39,19 \\
\hline Ensaio 8 & 36,58 \\
\hline Ensaio 7 & 17,03 \\
\hline Média & $\mathbf{4 6 , 9 9}$ \\
\hline
\end{tabular}

Tabela I. Índice de consistência acumulado dos 10 ensaios - relaxado.

\begin{tabular}{l|l}
\hline Ensaios & $\begin{array}{l}\text { Índice de consistência } \\
\text { (rígido) }\end{array}$ \\
\hline Ensaio 4 & 68,08 \\
\hline Ensaio 3 & 57,80 \\
\hline Ensaio 2 & 53,22 \\
\hline Ensaio 10 & 52,78 \\
\hline Ensaio 5 & 44,38 \\
\hline Ensaio 1 & 37,61 \\
\hline Ensaio 9 & 36,44 \\
\hline Ensaio 6 & 18,17 \\
\hline Ensaio 7 & 13,30 \\
\hline Ensaio 8 & 10,33 \\
\hline Média & $\mathbf{3 9 , 2 1}$ \\
\hline
\end{tabular}

Tabela II. Índice de consistência acumulado dos 10 ensaios - rígido.

Em relação à discrepância, o índice de consistência relaxado variou entre $17,03 \%$ e $68,8 \%$, com uma média de $46,99 \%$ de consistência entre as indexações dos livros (Tabela I). Já o índice de consistência rígido variou entre $10,33 \%$ e $68,08 \%$, com uma média de $39,21 \%$ (Tabela II).

Gil Leiva (2008) ressalta que a abordagem quantitativa da AI é de grande utilidade para avaliações periódicas em uma mesma unidade de informação por meio de ensaios de interconsistência, para comprovar discrepâncias entre a indexação do mesmo documento realizada por diferentes indexadores. Ademais, advoga que somente quando existir homogeneidade será possível aplicar as fórmulas ou equações para encontrar os índices de consistência e que a busca 
de um mesmo documento em vários catálogos de bibliotecas nos apresenta de que é bastante comum que não exista consenso nos assuntos designados.

Como explica o referido autor, os estudos sobre a AI com abordagem quantitativa revelam que os valores de consistência variam muito entre os indexadores, sendo que a média dos índices oscila entre os $25 \%$ e os $60 \%$ de coincidências obtidas na análise de assunto de um mesmo documento. Por outro lado, o estudo conduzido por Pinheiro (1978) revelou que os resultados apresentam variações entre 10\% e 80\%. De qualquer forma, os índices desta investigação ficaram dentro de ambas as margens.

Em comparação com alguns estudos publicados em Ciência da Informação, verifica-se que um índice de consistência alto é difícil de ser obtido, em especial quando o universo de pesquisa abarca diferentes instituições. Fora do contexto nacional, pode ser citado o estudo apresentado por Gil Leiva (1998), em que foi observado o índice de consistência em bibliotecas públicas na Espanha. Como resultado, o estudo demonstrou que o índice de consistência variava entre $46,6 \%$ no relaxado e $37,7 \%$ no rígido. No Brasil, Tartarotti (2014) comparou a representação temática realizada pelo bibliotecário em catálogo coletivo online e em base de dados de teses da área de Saúde, pautando-se na modalidade Avaliação Intrínseca Quantitativa ou Intrabibliotecário. De acordo com os resultados provenientes neste estudo acerca da aplicação da Fórmula de Hopper (1965) adaptada por Gil Leiva (2008) e da Fórmula de Rolling (1981), o índice de intraconsistência oscilou entre 40\% e 45\%.

No estudo realizado por Rubi, Gil Leiva e Fujita (2008), no qual foi observado o índice de consistência entre 30 bibliotecas universitárias localizadas nas Regiões do Sudeste e Sul do Brasil, por meio da fórmula matemática variante da fórmula de Hooper, apresentada anteriormente (Gil Leiva, 2008), o índice de consistência relaxado foi de 34,4 e o índice de consistência rígido fícou em 27,3 , demonstrando que a coincidência na determinação dos assuntos neste estudo não foi muito elevada.

Na concepção dos autores do estudo em destaque, a consistência da indexação está ligada ao desempenho do indexador e à qualidade das linguagens de indexação utilizadas como instrumentos de representação de assuntos. No geral, indicam que as causas para as diferenças significativas entre as indexações das bibliotecas universitárias analisadas da Região Sul e Sudeste se deve: à falta de compatibilidade entre os instrumentos utilizados, pois as bibliotecas, além de utilizarem linguagens distintas, empregam linguagens traduzidas de outros países, fator que impede que a cultura e características das bibliotecas nacionais sejam consideradas nestes instrumentos; uma política de indexação insuficiente ou inexistente, o que contribui diretamente para a falta de sistematização dos procedimentos de indexação e, por consequência, acarreta prejuízos e dispares na representação dos documentos; por fim, de que a inexistência de avaliação e divulgação de resultados da recuperação da informação contribui negativamente com o índice de consistência entre indexadores de diferentes bibliotecas.

No que tange à linguagem de indexação utilizada para a atribuição dos assuntos nos catálogos coletivos online das 9 bibliotecas federais da Região Nordeste participantes deste estudo, verifica-se que nenhuma biblioteca possui um vocabulário próprio elaborado por suas instituições. De forma geral, utilizam como principais linguagens de indexação: a Library of Congress Subject Headings (LCSH); a Terminologia de Assuntos da Biblioteca Nacional; e os assuntos tópicos da Lista de Autoridades da Rede Pergamum, constituída pelas instituições usuárias do software Pergamum, da qual as bibliotecas participam (que tem como base estas mesmas linguagens). Por decorrência, a ausência de uma linguagem própria e única para a tradução dos assuntos durante o processo de indexação pode ser apontada como um dos fatores para os baixos índices de consistência no processo de indexação.

Estes estudos fornecem uma indicação geral de que a consistência na indexação varia consideravelmente, sendo altamente improvável de ser alcançado 100\% de consistência (Hughes, 2011). Desse modo, os índices de consistência de vários estudos devem ser considerados separadamente, pois os valores de consistência dependem das condições em que a indexação foi realizada, como, por exemplo, as diferentes fórmulas adotadas (Tonta, 1991).

\section{ALGUMAS CONSIDERAÇÕES}

Ainda são muitos os fatores que cercam e influenciam a qualidade da indexação, sendo necessário que estes desafios sejam trabalhados com foco basilar na consistência da indexação. No âmbito das bibliotecas universitárias, a eficiente recuperação nos catálogos coletivos online está intimamente relacionada com a qualidade do processo de indexação. Dentre outros fatores, a qualidade da indexação possui relação direta com a política de indexação e a linguagem de indexação. 
Para o estabelecimento da política de indexação, por exemplo, como um guia para tomada de decisões, diversos são os elementos a serem considerados, pois cada biblioteca universitária possui suas próprias especificidades e características. Ademais, em bibliotecas universitárias estas diretrizes precisam ser descritas de maneira objetiva e estarem disponíveis aos atores do sistema de informação: dirigentes, bibliotecários e usuários. Como elemento mediador entre a linguagem do conteúdo do documento e a linguagem do usuário do sistema de recuperação de informação, a linguagem de indexação tem papel fundamental. Sendo tal sua importância, é necessário que cada biblioteca universitária disponha de uma linguagem de indexação própria, conferindo maior credibilidade da representatividade e visibilidade do sistema.

Apesar de sua importância teórica e aplicada, a AI ainda é uma temática pouco trabalhada na perspectiva teórica e aplicada da área. No Brasil, ainda são incipientes investigações preocupadas com a análise qualitativa e quantitativa em torno da AI, visando à melhoria dos índices de consistência que são reflexo de uma indexação de qualidade, principalmente no contexto institucional de bibliotecas universitárias.

Diante desta lacuna no campo científico da Ciência da Informação, a presente discussão foi conduzida de modo a contribuir para a melhoria dos produtos e serviços informacionais gerados no contexto de bibliotecas universitárias e despertar olhares sobre a questão. Os dados apresentados nesta pesquisa favorecem a composição do panorama atual da consistência da indexação na Região Nordeste do país. De modo efetivo, o resultado obtido com o índice de consistência das bibliotecas universitárias nesta região foi melhor em comparação com os dados apresentados pelas bibliotecas universitárias da Região Sul e Sudeste do Brasil.

A partir destes resultados, considera-se relevante a condução de pesquisas que avaliem outras regiões do país, a fim de traçar um panorama atual e mais amplo da qualidade do processo de indexação em sistemas de recuperação de informação das bibliotecas universitárias no contexto brasileiro, bem como para o enriquecimento de discussões complementares na temática.

\section{BIBLIOGRAFIA}

ABAD GARCÍA, M.F.; ABAD PÉREZ, I.A. e BENAVENT, A. Evaluación de la consistencia en la indización del repertorio documentación médica española. Revista Española de Documentación Científica, 1998, vol. 21, nº 4, p. 389-401.

CHOI, Y. Usefulness of social tagging in organizing and providing access to the web: an analysis of indexing consistency and quality. Dissertation, Doctor of Philosophy in Library and Information Science, University of Illinois at Urbana, Champaign, 2011.

COOPER, W.S. Is interindexer consistency a hobgoblin? American Documentation, 1969, vol. 20, nº 3, p. $268-278$.

FUNK, M.E.; REID, C.A. e McGOOGAN, L.S. Indexing consistency in Medline. Bulletin of the Medical Library Association, 1983, vol. 71, n 2, p. 176-183.

FURNAS, G.W. et al. The vocabulary problem in human-system communication. Communications of the ACM, 1987, vol. 30, nº 11, p. 964-971.

GIL LEIVA, I. Manual de indización: teoría y práctica. Gijón: Trea, 2008.

GIL LEIVA, I.; RUBI, M.P. e FUJITA, M.S.L. Consistência na indexação em bibliotecas universitárias brasileiras. Transinformação, set./dez. 2008, vol. 20, no 3, p. 233-253.

HOOPER, R.S. Indexer consistency tests: origin, measurement, results, and utilization. Bethesda: IBM Coporation, 1965.

HUGHES, A.V. Inter-indexer consistency in graphic materials indexing at the National Library of Wales. Journal of Documentation, 2011, vol. 67, $\mathrm{n}^{\circ}$ 1, p. 9-32.

INÁCIO, M. de O. Avaliação da indexação em bibliotecas universitárias: uma aplicação em catálogos online. Dissertação, Mestrado em Ciência da Informação, Faculdade de Filosofia e Ciências, Universidade Estadual Paulista, Marília, 2012.

LEININGER, K. Interindexer consistency in PsycINFO. Journal of Librarianship and Information Science, mar. 2000, vol. 32, no 1, p. 4-8.

LANCASTER, F.W. Indexação e resumos: teoria e prática. 2. ed. Brasília: Briquet de Lemos, 2004.

LEONARD, L.E. Inter-indexer consistency studies, 1954-1975: a review of the literature and summary of study results. Occasional Papers, ${ }^{\circ}$ 31, Graduate School of Library Science, University of Illinois, Urbana, IL. 1977.

OTERO, A.M. Exame filosófico do vocabulário sociológico. En: M. BUNGE. Epistemologia: curso de atualização. São Paulo: T. A. Queiroz; Editora da Universidade de São Paulo, 1980, p. 139-165.

PAEK, T. e CHANDRASEKAR, R. Windows as a second language: an overview of the jargon project. Proceedings of the First International Conference on Augmented Cognition, 2005. 
PINHEIRO, L.V.R. Medidas de consistência da indexação: interconsistência. Ciência da Informação, 1978, vol. 7, nº 2, p. 109-114.

ROLLING, L. Indexing consistency, quality and efficiency. Information Processing \& Management, 1981, vol. 17, p. 69-76.

SAARTI, J. Consistency of subject indexing of novels by public library professionals and patrons, Journal of Documentation, 2002, vol. 58, $\mathrm{n}^{\circ}$ 1, p. 49-65.

TARTAROTTI, R.C.D. Atuação bibliotecária no tratamento temático da informação em unidades informacionais: um estudo comparativo qualitativo-quantitativo. Dissertação, Mestrado em Ciência, Tecnologia e Sociedade, Universidade Federal de São Carlos, São Carlos, 2014.

TONTA, Y. A study of indexing consistency between Library of Congress and British Library catalogers. Library Resources \& Technical Services, 1991, vol. 35, p. 177-185.

WOLFRAM, D.; OLSON, H.A. e BLOOM, R. Measuring consistency for multiple taggers using vector space modeling. Journal of The American Society for Information Science and Technology, 2009, vol. 60, $\mathrm{n}^{\circ}$ 10, p. 19952003.

ZUNDE, P. e DEXTER, M.E. Indexing consistency and quality. American Documentation, 1969, vol. 20, nº 3, p. 259 267. 


\section{APÊNDICES}

ENSAIO 1

Livro 1: Angelo, J.G.; Fattini, C.A. Anatomia humana sistêmica e segmentar. 3.ed. São Paulo: Atheneu, 2007.

Quadro III. Levantamento dos assuntos catalogados do mesmo livro nos catálogos coletivos online - Livro 1.

\begin{tabular}{|l|l|c|}
\hline Sigla da Biblioteca & Assuntos & Classificação \\
\hline UFAL & $\begin{array}{l}\text { Anatomia humana - } \\
\text { Estudo e ensino }\end{array}$ & 611 \\
\hline UFBA & Anatomia humana & $611.1 / 9$ \\
\hline UFC & Anatomia humana & 611 \\
\hline UFMA & Anatomia humana & 611 \\
\hline UFPB & $\begin{array}{l}\text { Anatomia humana } \\
\text { Anatomia }\end{array}$ & 611 \\
\hline UFPE & Anatomia humana & 611 \\
\hline UFPI & Anatomia & 611 \\
\hline UFRN & Anatomia humana & 611 \\
\hline UFS & $\begin{array}{l}\text { Anatomia humana } \\
\text { Medicina } \\
\text { Coluna vertebral } \\
\text { Pescoço } \\
\text { Sistema cardiovascular } \\
\text { Aparelho digestivo } \\
\text { Sistema endócrino } \\
\text { Sistema } \\
\text { musculoesquelético } \\
\text { Aparelho geniturinário } \\
\text { Cabeça } \\
\text { Aparelho respiratório }\end{array}$ & 611 \\
\hline
\end{tabular}

Tabela III. Índices de consistência entre pares de bibliotecas - Livro 1.

\begin{tabular}{|c|c|c|}
\hline $\begin{array}{l}\text { Pares de } \\
\text { Bibliotecas }\end{array}$ & $\begin{array}{l}\text { Índice de } \\
\text { consistência } \\
\text { (relaxado) }\end{array}$ & $\begin{array}{l}\text { Índice de } \\
\text { consistência } \\
\text { (rígido) }\end{array}$ \\
\hline UFAL e UFBA & 33 & 0 \\
\hline UFAL e UFC & 33 & 0 \\
\hline UFAL e UFMA & 33 & 0 \\
\hline UFAL e UFPB & 20 & 0 \\
\hline UFAL e UFPE & 33 & 0 \\
\hline UFAL e UFPI & 33 & 0 \\
\hline UFAL e UFRN & 33 & 0 \\
\hline UFAL e UFS & 4 & 0 \\
\hline UFBA e UFC & 100 & 100 \\
\hline UFBA e UFMA & 100 & 100 \\
\hline UFBA e UFPB & 50 & 50 \\
\hline UFBA e UFPE & 100 & 100 \\
\hline UFBA e UFPI & 33 & 0 \\
\hline UFBA e UFRN & 100 & 100 \\
\hline UFBA e UFS & 9 & 9 \\
\hline UFC e UFMA & 100 & 100 \\
\hline UFC e UFPB & 50 & 50 \\
\hline UFC e UFPE & 100 & 100 \\
\hline UFC e UFPI & 33 & 0 \\
\hline UFC e UFRN & 100 & 100 \\
\hline UFC e UFS & 9 & 9 \\
\hline UFMA e UFPB & 50 & 50 \\
\hline UFMA e UFPE & 100 & 100 \\
\hline UFMA e UFPI & 33 & 0 \\
\hline UFMA e UFRN & 100 & 100 \\
\hline UFMA e UFS & 4 & 9 \\
\hline UFPB e UFPE & 50 & 50 \\
\hline UFPB e UFPI & 50 & 50 \\
\hline UFPB e UFRN & 50 & 50 \\
\hline UFPB e UFS & 9 & 9 \\
\hline UFPE e UFPI & 33 & 0 \\
\hline UFPE e UFRN & 100 & 100 \\
\hline UFPE e UFS & 9 & 9 \\
\hline UFPI e UFRN & 33 & 0 \\
\hline UFPI e UFS & 4 & 0 \\
\hline UFRN e UFS & 9 & 9 \\
\hline Média & 48,33 & 37,61 \\
\hline
\end{tabular}




\section{ENSAIO 2}

Livro 2: Griffiths, A.J.F; Suzuki, D.T. e Miller, J.H. Introdução à genética. 9.ed. Rio de Janeiro: Guanabara Koogan, 2008.

Quadro IV. Levantamento dos assuntos catalogados do mesmo livro nos catálogos coletivos online - Livro 2.

\begin{tabular}{|l|l|c|}
\hline Sigla da Biblioteca & Assunto & Classificação \\
\hline UFAL & $\begin{array}{l}\text { Genética } \\
\text { Hereditariedade }\end{array}$ & 575 \\
\hline UFBA & $\begin{array}{l}\text { Genética } \\
\text { Hereditariedade }\end{array}$ & 575 \\
\hline UFC & $\begin{array}{l}\text { Genética } \\
\text { Hereditariedade }\end{array}$ & 575.1 \\
\hline UFMA & $\begin{array}{l}\text { Hereditariedade } \\
\text { Genética }\end{array}$ & 575 \\
\hline UFPB & $\begin{array}{l}\text { Genética } \\
\text { Hereditariedade }\end{array}$ & 575 \\
\hline UFPE & $\begin{array}{l}\text { Genética } \\
\text { Genética humana }\end{array}$ & 575.1 \\
\hline UFPI & Genética & 575 \\
\hline UFRN & $\begin{array}{l}\text { Genética humana } \\
\text { Herança monogênica } \\
\text { DNA } \\
\text { Alterações cromossômicas } \\
\text { Genética de populações }\end{array}$ & \\
\hline UFS & $\begin{array}{l}\text { Genética } \\
\text { Biologia }\end{array}$ \\
\hline
\end{tabular}

Tabela IV. Índices de consistência entre pares de bibliotecas - Livro 2.

\begin{tabular}{|c|c|c|}
\hline $\begin{array}{l}\text { Pares de } \\
\text { Bibliotecas }\end{array}$ & $\begin{array}{l}\text { Índice de } \\
\text { consistência } \\
\text { (relaxado) }\end{array}$ & $\begin{array}{l}\text { Índice de } \\
\text { consistência } \\
\text { (rígido) }\end{array}$ \\
\hline UFAL e UFBA & 100 & 100 \\
\hline UFAL e UFC & 100 & 100 \\
\hline UFAL e UFMA & 100 & 100 \\
\hline UFAL e UFPB & 100 & 100 \\
\hline UFAL e UFPE & 50 & 50 \\
\hline UFAL e UFPI & 50 & 50 \\
\hline UFAL e UFRN & 8 & 0 \\
\hline UFAL e UFS & 50 & 50 \\
\hline UFBA e UFC & 100 & 100 \\
\hline UFBA e UFMA & 100 & 100 \\
\hline UFBA e UFPB & 100 & 100 \\
\hline UFBA e UFPE & 50 & 50 \\
\hline UFBA e UFPI & 50 & 50 \\
\hline UFBA e UFRN & 8 & 0 \\
\hline UFBA e UFS & 50 & 50 \\
\hline UFC e UFMA & 100 & 100 \\
\hline UFC e UFPB & 100 & 100 \\
\hline UFC e UFPE & 50 & 50 \\
\hline UFC e UFPI & 50 & 50 \\
\hline UFC e UFRN & 8 & 0 \\
\hline UFC e UFS & 50 & 50 \\
\hline UFMA e UFPB & 100 & 100 \\
\hline UFMA e UFPE & 50 & 50 \\
\hline UFMA e UFPI & 50 & 50 \\
\hline UFMA e UFRN & 8 & 0 \\
\hline UFMA e UFS & 50 & 50 \\
\hline UFPB e UFPE & 50 & 50 \\
\hline UFPB e UFPI & 50 & 50 \\
\hline UFPB e UFRN & 8 & 0 \\
\hline UFPB e UFS & 50 & 50 \\
\hline UFPE e UFPI & 50 & 50 \\
\hline UFPE e UFRN & 27 & 16 \\
\hline UFPE e UFS & 50 & 50 \\
\hline UFPI e UFRN & 9 & 0 \\
\hline UFPI e UFS & 50 & 50 \\
\hline UFRN e UFS & 8 & 0 \\
\hline Média & 55,11 & 53,22 \\
\hline
\end{tabular}




\section{ENSAIO 3}

Livro 3: Goodman, L.S. et al. As bases farmacológicas da terapêutica. 11.ed. Rio de Janeiro: McGraw-Hill, 2006.

Quadro V. Levantamento dos assuntos catalogados do mesmo livro nos catálogos coletivos online - Livro 3.

\begin{tabular}{|l|l|c|}
\hline Sigla da Biblioteca & Assunto & Classificação \\
\hline UFAL & $\begin{array}{l}\text { Farmacologia } \\
\text { Terapêtica }\end{array}$ & 615 \\
\hline UFBA & $\begin{array}{l}\text { Farmacologia } \\
\text { Terapêutica }\end{array}$ & 615 \\
\hline UFC & $\begin{array}{l}\text { Farmacologia } \\
\text { Terapêutica } \\
\text { Quimioterapia }\end{array}$ & 615.1 \\
\hline UFMA & $\begin{array}{l}\text { Farmacologia } \\
\text { Quimioterapia }\end{array}$ & 615.03 \\
\hline UFPB & $\begin{array}{l}\text { Terapêutica } \\
\text { Farmacognética } \\
\text { Fármacos } \\
\text { Hormônios } \\
\text { Quimioterapia }\end{array}$ & 615 \\
\hline UFPE & $\begin{array}{l}\text { Farmacologia } \\
\text { Terapêutica } \\
\text { Toxicologia }\end{array}$ & 615.03 \\
\hline UFPI & $\begin{array}{l}\text { Terapeutica } \\
\text { Farmacologia }\end{array}$ & 615.1 \\
\hline UFRN & $\begin{array}{l}\text { Farmacologia } \\
\text { Terapêutica } \\
\text { Toxicologia }\end{array}$ & 615 \\
\hline UFS & $\begin{array}{l}\text { Farmacologia } \\
\text { Terapêutica }\end{array}$ & 615 \\
\hline
\end{tabular}

Tabela V. Índices de consistência entre pares de bibliotecas - Livro 3 .

\begin{tabular}{|c|c|c|}
\hline $\begin{array}{l}\text { Pares de } \\
\text { Bibliotecas }\end{array}$ & $\begin{array}{l}\text { Índice de } \\
\text { consistência } \\
\text { (relaxado) }\end{array}$ & $\begin{array}{l}\text { Índice de } \\
\text { consistência } \\
\text { (rígido) }\end{array}$ \\
\hline UFAL e UFBA & 100 & 100 \\
\hline UFAL e UFC & 66 & 66 \\
\hline UFAL e UFMA & 50 & 50 \\
\hline UFAL e UFPB & 16 & 16 \\
\hline UFAL e UFPE & 66 & 66 \\
\hline UFAL e UFPI & 100 & 100 \\
\hline UFAL e UFRN & 66 & 66 \\
\hline UFAL e UFS & 100 & 100 \\
\hline UFBA e UFC & 66 & 66 \\
\hline UFBA e UFMA & 50 & 50 \\
\hline UFBA e UFPB & 16 & 16 \\
\hline UFBA e UFPE & 66 & 66 \\
\hline UFBA e UFPI & 100 & 100 \\
\hline UFBA e UFRN & 66 & 66 \\
\hline UFBA e UFS & 100 & 100 \\
\hline UFC e UFMA & 66 & 66 \\
\hline UFC e UFPB & 33 & 33 \\
\hline UFC e UFPE & 66 & 66 \\
\hline UFC e UFPI & 66 & 66 \\
\hline UFC e UFRN & 66 & 66 \\
\hline UFC e UFS & 66 & 66 \\
\hline UFMA e UFPB & 16 & 16 \\
\hline UFMA e UFPE & 25 & 25 \\
\hline UFMA e UFPI & 50 & 50 \\
\hline UFMA e UFRN & 25 & 25 \\
\hline UFMA e UFS & 50 & 50 \\
\hline UFPB e UFPE & 14 & 14 \\
\hline UFPB e UFPI & 16 & 16 \\
\hline UFPB e UFRN & 14 & 14 \\
\hline UFPB e UFS & 16 & 16 \\
\hline UFPE e UFPI & 66 & 66 \\
\hline UFPE e UFRN & 100 & 100 \\
\hline UFPE e UFS & 66 & 66 \\
\hline UFPI e UFRN & 66 & 66 \\
\hline UFPI e UFS & 100 & 100 \\
\hline UFRN e UFS & 66 & 66 \\
\hline Média & 57,80 & 57,80 \\
\hline
\end{tabular}




\section{ENSAIO 4}

Livro 4: Levinson, W.E. e Kyaw, M.M. Microbiologia médica e imunologia. 10.ed. Porto Alegre, RS: Artmed, 2010.

Quadro VI. Levantamento dos assuntos catalogados do mesmo livro nos catálogos coletivos online - Livro 4.

\begin{tabular}{|l|l|l|}
\hline Sigla da Biblioteca & Assunto & Classificação \\
\hline UFAL & $\begin{array}{l}\text { Imunologia } \\
\text { Microbiologia } \\
\text { Microbiologia médica }\end{array}$ & 579 \\
\hline UFBA & $\begin{array}{l}\text { Microbiologia médica } \\
\text { Imunologia }\end{array}$ & 579.61 \\
\hline UFC & $\begin{array}{l}\text { Microbiologia médica } \\
\text { Imunologia } \\
\text { Bacteriologia } \\
\text { Alergia e Imunologia }\end{array}$ & 616.01 \\
\hline UFMA & $\begin{array}{l}\text { Microbiologia médica } \\
\text { Imunologia }\end{array}$ & $616-022.7$ \\
\hline UFPB & $\begin{array}{l}\text { Microbiologia médica } \\
\text { Imunologia }\end{array}$ & $579+612.017$ \\
\hline UFPE & $\begin{array}{l}\text { Microbiologia médica } \\
\text { Imunologia }\end{array}$ & 616.01 \\
\hline UFPI & $\begin{array}{l}\text { Microbiologia médica } \\
\text { Imunologia }\end{array}$ & 616.01 \\
\hline UFRN & $\begin{array}{l}\text { Microbiologia médica } \\
\text { Imunologia }\end{array}$ & $616-093 /-098$ \\
\hline UFS & $\begin{array}{l}\text { Microbiologia médica } \\
\text { Imunologia } \\
\text { Sistema imunológico } \\
\text { Anatomia humana } \\
\text { Corpo humano } \\
\text { Parasitologia } \\
\text { Micologia }\end{array}$ & $579.61: 612.017$ \\
\hline
\end{tabular}

Tabela VI. Indices de consistência entre pares de bibliotecas - Livro 4 .

\begin{tabular}{|c|c|c|}
\hline $\begin{array}{l}\text { Pares de } \\
\text { Bibliotecas }\end{array}$ & $\begin{array}{l}\text { Índice de } \\
\text { consistência } \\
\text { (relaxado) }\end{array}$ & $\begin{array}{l}\text { Índice de } \\
\text { consistência } \\
\text { (rígido) }\end{array}$ \\
\hline UFAL e UFBA & 66 & 66 \\
\hline UFAL e UFC & 40 & 40 \\
\hline UFAL e UFMA & 66 & 66 \\
\hline UFAL e UFPB & 66 & 66 \\
\hline UFAL e UFPE & 66 & 66 \\
\hline UFAL e UFPI & 66 & 66 \\
\hline UFAL e UFRN & 66 & 66 \\
\hline UFAL e UFS & 25 & 25 \\
\hline UFBA e UFC & 50 & 50 \\
\hline UFBA e UFMA & 100 & 100 \\
\hline UFBA e UFPB & 100 & 100 \\
\hline UFBA e UFPE & 100 & 100 \\
\hline UFBA e UFPI & 100 & 100 \\
\hline UFBA e UFRN & 100 & 100 \\
\hline UFBA e UFS & 28 & 28 \\
\hline UFC e UFMA & 50 & 50 \\
\hline UFC e UFPB & 50 & 50 \\
\hline UFC e UFPE & 50 & 50 \\
\hline UFC e UFPI & 50 & 50 \\
\hline UFC e UFRN & 50 & 50 \\
\hline UFC e UFS & 22 & 22 \\
\hline UFMA e UFPB & 100 & 100 \\
\hline UFMA e UFPE & 100 & 100 \\
\hline UFMA e UFPI & 100 & 100 \\
\hline UFMA e UFRN & 100 & 100 \\
\hline UFMA e UFS & 28 & 28 \\
\hline UFPB e UFPE & 100 & 100 \\
\hline UFPB e UFPI & 100 & 100 \\
\hline UFPB e UFRN & 100 & 100 \\
\hline UFPB e UFS & 28 & 28 \\
\hline UFPE e UFPI & 100 & 100 \\
\hline UFPE e UFRN & 100 & 100 \\
\hline UFPE e UFS & 28 & 28 \\
\hline UFPI e UFRN & 100 & 100 \\
\hline UFPI e UFS & 28 & 28 \\
\hline UFRN e UFS & 28 & 28 \\
\hline Média & 68,08 & 68,08 \\
\hline
\end{tabular}




\section{ENSAIO 5}

Livro 5: Rey, L. Bases da parasitologia médica. 3.ed. Rio de Janeiro: Guanabara Koogan, 2010.

Quadro VII. Levantamento dos assuntos catalogados do mesmo livro nos catálogos coletivos online - Livro 5.

\begin{tabular}{|l|l|c|}
\hline Sigla da Biblioteca & Assunto & Classificação \\
\hline UFAL & $\begin{array}{l}\text { Medicina } \\
\text { Parasitologia médica } \\
\text { Imunologia } \\
\text { Biologia molecular } \\
\text { Quimioterapia } \\
\text { Epidemiologia }\end{array}$ & 616.99 \\
\hline UFBA & Parasitologia médica & \\
\hline UFC & $\begin{array}{l}\text { Parasitologia médica } \\
\text { Parasitologia }\end{array}$ & 676.8 \\
\hline UFMA & Parasitologia médica & 616.96 \\
\hline UFPB & Parasitologia médica & 576.8 \\
\hline UFPE & $\begin{array}{l}\text { Parasitologia } \\
\text { Parasitologia médica }\end{array}$ & 616.96 \\
\hline UFPI & Parasitologia médica & 616.96 \\
\hline UFRN & $\begin{array}{l}\text { Doenças parasitárias } \\
\text { Parasitologia } \\
\text { Medicina } \\
\text { Parasitologia médica } \\
\text { Infecções parasitárias }\end{array}$ & 616.99 \\
\hline UFS & $\begin{array}{l}\text { Parasitologia médica } \\
\text { Medicina } \\
\text { Diagnóstico parasitológico } \\
\text { Doenças parasitárias }\end{array}$ & $616-022$ \\
\hline
\end{tabular}

Tabela VII. Índices de consistência entre pares de bibliotecas - Livro 5.

\begin{tabular}{|c|c|c|}
\hline $\begin{array}{l}\text { Pares de } \\
\text { Bibliotecas }\end{array}$ & $\begin{array}{l}\text { Índice de } \\
\text { consistência } \\
\text { (relaxado) }\end{array}$ & $\begin{array}{l}\text { Índice de } \\
\text { consistência } \\
\text { (rígido) }\end{array}$ \\
\hline UFAL e UFBA & 16 & 16 \\
\hline UFAL e UFC & 16 & 16 \\
\hline UFAL e UFMA & 16 & 16 \\
\hline UFAL e UFPB & 16 & 16 \\
\hline UFAL e UFPE & 16 & 16 \\
\hline UFAL e UFPI & 16 & 16 \\
\hline UFAL e UFRN & 22 & 22 \\
\hline UFAL e UFS & 25 & 25 \\
\hline UFBA e UFC & 50 & 50 \\
\hline UFBA e UFMA & 100 & 100 \\
\hline UFBA e UFPB & 100 & 100 \\
\hline UFBA e UFPE & 50 & 50 \\
\hline UFBA e UFPI & 100 & 100 \\
\hline UFBA e UFRN & 20 & 20 \\
\hline UFBA e UFS & 25 & 25 \\
\hline UFC e UFMA & 50 & 50 \\
\hline UFC e UFPB & 50 & 50 \\
\hline UFC e UFPE & 100 & 100 \\
\hline UFC e UFPI & 50 & 50 \\
\hline UFC e UFRN & 40 & 40 \\
\hline UFC e UFS & 20 & 20 \\
\hline UFMA e UFPB & 100 & 100 \\
\hline UFMA e UFPE & 50 & 50 \\
\hline UFMA e UFPI & 100 & 100 \\
\hline UFMA e UFRN & 20 & 20 \\
\hline UFMA e UFS & 25 & 25 \\
\hline UFPB e UFPE & 50 & 50 \\
\hline UFPB e UFPI & 100 & 100 \\
\hline UFPB e UFRN & 20 & 20 \\
\hline UFPB e UFS & 52 & 25 \\
\hline UFPE e UFPI & 50 & 50 \\
\hline UFPE e UFRN & 40 & 40 \\
\hline UFPE e UFS & 20 & 20 \\
\hline UFPI e UFRN & 25 & 25 \\
\hline UFPI e UFS & 25 & 25 \\
\hline UFRN e UFS & 50 & 50 \\
\hline Média & 44,38 & 44,38 \\
\hline
\end{tabular}




\section{ENSAIO 6}

Livro 6: Chiavenato, I. Administração: teoria, processo e prática. 4.ed. Ver. E atual. Rio de Janeiro: Elsevier, 2007.

Quadro VIII. Levantamento dos assuntos catalogados do mesmo livro nos catálogos coletivos online - Livro 6

\begin{tabular}{|l|l|l|}
\hline Sigla da Biblioteca & Assunto & Classificação \\
\hline UFAL & $\begin{array}{l}\text { Administração de empresas } \\
\text { Administração }\end{array}$ & 658 \\
\hline UFBA & Administração de empresas & 658 \\
\hline UFC & Administração & 658 \\
\hline UFMA & Administração - Teoria & 65.01 \\
\hline UFPB & $\begin{array}{l}\text { Administração de empresas } \\
\text { Planejamento estratégico } \\
\text { Planejamento tático }\end{array}$ & 658 \\
\hline UFPE & $\begin{array}{l}\text { Administração de empresas } \\
\text { Administração - Teoria }\end{array}$ & 658.001 \\
\hline UFPI & $\begin{array}{l}\text { Empresas - Administração } \\
\text { Administração - Teoria } \\
\text { Administração }\end{array}$ & 658 \\
\hline UFRN & $\begin{array}{l}\text { Teoria da Administração } \\
\text { Planejamento empresarial } \\
\text { Administração de empresas }\end{array}$ & 658 \\
\hline UFS & $\begin{array}{l}\text { Administração de empresas } \\
\text { Teoria da administração } \\
\text { Planejamento estratégico } \\
\text { Planejamento empresarial } \\
\text { Gerência } \\
\text { Organização e métodos }\end{array}$ & 658 \\
\hline
\end{tabular}

Tabela VIII. Índices de consistência entre pares de bibliotecas - Livro 6.

\begin{tabular}{|c|c|c|}
\hline $\begin{array}{l}\text { Pares de } \\
\text { Bibliotecas }\end{array}$ & $\begin{array}{l}\text { Índice de } \\
\text { consistência } \\
\text { (relaxado) }\end{array}$ & $\begin{array}{l}\text { Índice de } \\
\text { consistência } \\
\text { (rígido) }\end{array}$ \\
\hline UFAL e UFBA & 50 & 50 \\
\hline UFAL e UFC & 50 & 50 \\
\hline UFAL e UFMA & 25 & 0 \\
\hline UFAL e UFPB & 25 & 25 \\
\hline UFAL e UFPE & 50 & 50 \\
\hline UFAL e UFPI & 50 & 25 \\
\hline UFAL e UFRN & 25 & 0 \\
\hline UFAL e UFS & 21 & 14 \\
\hline UFBA e UFC & 50 & 0 \\
\hline UFBA e UFMA & 50 & 0 \\
\hline UFBA e UFPB & 33 & 33 \\
\hline UFBA e UFPE & 50 & 50 \\
\hline UFBA e UFPI & 66 & 0 \\
\hline UFBA e UFRN & 50 & 33 \\
\hline UFBA e UFS & 25 & 16 \\
\hline UFC e UFMA & 50 & 0 \\
\hline UFC e UFPB & 16 & 0 \\
\hline UFC e UFPE & 50 & 0 \\
\hline UFC e UFPI & 50 & 33 \\
\hline UFC e UFRN & 33 & 0 \\
\hline UFC e UFS & 16 & 0 \\
\hline UFMA e UFPB & 16 & 0 \\
\hline UFMA e UFPE & 50 & 50 \\
\hline UFMA e UFPI & 66 & 33 \\
\hline UFMA e UFRN & 33 & 0 \\
\hline UFMA e UFS & 16 & 0 \\
\hline UFPB e UFPE & 37 & 25 \\
\hline UFPB e UFPI & 40 & 0 \\
\hline UFPB e UFRN & 50 & 16 \\
\hline UFPB e UFS & 37 & 25 \\
\hline UFPE e UFPI & 50 & 25 \\
\hline UFPE e UFRN & 37 & 25 \\
\hline UFPE e UFS & 29 & 14 \\
\hline UFPI e UFRN & 40 & 0 \\
\hline UFPI e UFS & 31 & 0 \\
\hline UFRN e UFS & 44 & 37 \\
\hline Média & 39,19 & 18,17 \\
\hline
\end{tabular}




\section{ENSAIO 7}

Livro 7: Maingueneau, D. Novas tendências em análise do discurso. 3.ed. Campinas, SP: Pontes: UNICAMP, 1997.

Quadro IX. Levantamento dos assuntos catalogados do mesmo livro nos catálogos coletivos online - Livro 7.

\begin{tabular}{|l|l|l|}
\hline Sigla da Biblioteca & Assunto & Classificação \\
\hline UFAL & Pragmática & 800.5 \\
\hline UFBA & $\begin{array}{l}\text { Análise do discurso } \\
\text { Linguagem } \\
\text { Retórica }\end{array}$ & $\begin{array}{l}801.7: 003 \\
82-5(042.5)\end{array}$ \\
\hline UFC & - & 401.41 \\
\hline UFMA & $\begin{array}{l}\text { Análise do discurso } \\
\text { Linguística }\end{array}$ & 801 \\
\hline UFPB & $\begin{array}{l}\text { Discursos } \\
\text { Análise do Discurso }\end{array}$ & $82-5$ \\
\hline UFPE & $\begin{array}{l}\text { Análise do Discurso } \\
\text { Linguística - Análise do } \\
\text { discurso } \\
\text { Linguística - Estudo e ensino }\end{array}$ & 810 \\
\hline UFPI & Discursos & 808.51 \\
\hline UFRN & $\begin{array}{l}\text { Análise do discurso } \\
\text { Literatura }\end{array}$ & $82-5$ \\
\hline UFS & $\begin{array}{l}\text { Linguística } \\
\text { Análise do Discurso } \\
\text { Linguagem e línguas }\end{array}$ & 81 '42 \\
\hline
\end{tabular}

Tabela IX. Índices de consistência entre pares de bibliotecas - Livro 7.

\begin{tabular}{|c|c|c|}
\hline $\begin{array}{l}\text { Pares de } \\
\text { Bibliotecas }\end{array}$ & $\begin{array}{l}\text { Índice de } \\
\text { consistência } \\
\text { (relaxado) }\end{array}$ & $\begin{array}{l}\text { Índice de } \\
\text { consistência } \\
\text { (rígido) }\end{array}$ \\
\hline UFAL e UFBA & 0 & 0 \\
\hline UFAL e UFC & 0 & 0 \\
\hline UFAL e UFMA & 0 & 0 \\
\hline UFAL e UFPB & 0 & 0 \\
\hline UFAL e UFPE & 0 & 0 \\
\hline UFAL e UFPI & 0 & 0 \\
\hline UFAL e UFRN & 0 & 0 \\
\hline UFAL e UFS & 0 & 0 \\
\hline UFBA e UFC & 0 & 0 \\
\hline UFBA e UFMA & 25 & 25 \\
\hline UFBA e UFPB & 25 & 25 \\
\hline UFBA e UFPE & 20 & 20 \\
\hline UFBA e UFPI & 17 & 0 \\
\hline UFBA e UFRN & 37 & 25 \\
\hline UFBA e UFS & 30 & 20 \\
\hline UFC e UFMA & 0 & 0 \\
\hline UFC e UFPB & 0 & 0 \\
\hline UFC e UFPE & 0 & 0 \\
\hline UFC e UFPI & 0 & 0 \\
\hline UFC e UFRN & 0 & 0 \\
\hline UFC e UFS & 0 & 0 \\
\hline UFMA e UFPB & 33 & 33 \\
\hline UFMA e UFPE & 50 & 25 \\
\hline UFMA e UFPI & 25 & 0 \\
\hline UFMA e UFRN & 33 & 33 \\
\hline UFMA e UFS & 50 & 50 \\
\hline UFPB e UFPE & 37 & 25 \\
\hline UFPB e UFPI & 50 & 50 \\
\hline UFPB e UFRN & 33 & 33 \\
\hline UFPB e UFS & 25 & 25 \\
\hline UFPE e UFPI & 33 & 0 \\
\hline UFPE e UFRN & 25 & 25 \\
\hline UFPE e UFS & 40 & 40 \\
\hline UFPI e UFRN & 0 & 0 \\
\hline UFPI e UFS & 0 & 0 \\
\hline UFRN e UFS & 25 & 25 \\
\hline Média & 17,03 & 13,30 \\
\hline
\end{tabular}




\section{ENSAIO 8}

Livro 8: AQUINO, M.F. de. O conceito de religião em Hegel. São Paulo: Loyola, 1989.

Quadro X. Levantamento dos assuntos catalogados do mesmo livro nos catálogos coletivos online - Livro 8.

\begin{tabular}{|l|l|l|}
\hline Sigla da Biblioteca & Assunto & Classificação \\
\hline UFAL & $\begin{array}{l}\text { Religião x Filosofia } \\
\text { Filosofia e religião } \\
\text { Fenomenologia } \\
\text { Lógica } \\
\text { Filosofia alemã }\end{array}$ & 130.121 \\
Religião x Filosofia & \\
\hline UFBA & $\begin{array}{l}\text { Filosofia e religião } \\
\text { Fenomenologia }\end{array}$ & 130.32 \\
\hline UFC & $\begin{array}{l}\text { Religião - Hegel } \\
\text { Filosofia - Hegel }\end{array}$ & 200.1 \\
\hline UFMA & $\begin{array}{l}\text { Hegel, Wilhelm Friedrich, } \\
\text { 1770-1831 } \\
\text { X Crítica e interpretação } \\
\text { Filosofia e religião } \\
\text { Religião x Filosofia } \\
\text { Lógica } \\
\text { Fenomenologia }\end{array}$ & 141 \\
\hline UFPB & $\begin{array}{l}\text { Religião x Filosofia } \\
\text { Hegel, Wilhelm Friedrich, 1770- } \\
1831\end{array}$ & \\
Contribuições em religião & 291.1 \\
\hline UFPE & $\begin{array}{l}\text { Filósofos Alemães } \\
\text { Religião - Filosofia e teorias } \\
\text { Hegel, Wilhelm Friedrich, } \\
1770-1831\end{array}$ & 200.1 \\
X Crítica e interpretação & \\
\hline UFPI & $\begin{array}{l}\text { Filosofia do espírito } \\
\text { Fenomenologia } \\
\text { Filosofia e religião } \\
\text { Hegel, Wilhelm Friedrich, } \\
1770-1831\end{array}$ & 13 \\
Filosofia & $\begin{array}{l}\text { Arte } \\
\text { Psicologia } \\
\text { Metodologia } \\
\text { Lógica } \\
\text { Religião x Filosofia } \\
\text { Filosofia }\end{array}$ & \\
\hline UFR & & 1 \\
\hline
\end{tabular}

Tabela X. Índices de consistência entre pares de bibliotecas - Livro 8.

\begin{tabular}{|c|c|c|}
\hline $\begin{array}{l}\text { Pares de } \\
\text { Bibliotecas }\end{array}$ & $\begin{array}{l}\text { Índice de } \\
\text { consistência } \\
\text { (relaxado) }\end{array}$ & $\begin{array}{l}\text { Índice de } \\
\text { consistência } \\
\text { (rígido) }\end{array}$ \\
\hline UFAL e UFBA & 20 & 20 \\
\hline UFAL e UFC & 58 & 33 \\
\hline UFAL e UFMA & 42 & 0 \\
\hline UFAL e UFPB & 44 & 44 \\
\hline UFAL e UFPE & 17 & 17 \\
\hline UFAL e UFPI & 36 & 0 \\
\hline UFAL e UFRN & 33 & 22 \\
\hline UFAL e UFS & 35 & 10 \\
\hline UFBA e UFC & 50 & 0 \\
\hline UFBA e UFMA & 50 & 0 \\
\hline UFBA e UFPB & 20 & 20 \\
\hline UFBA e UFPE & 50 & 50 \\
\hline UFBA e UFPI & 33 & 0 \\
\hline UFBA e UFRN & 40 & 0 \\
\hline UFBA e UFS & 25 & 17 \\
\hline UFC e UFMA & 33 & 0 \\
\hline UFC e UFPB & 50 & 33 \\
\hline UFC e UFPE & 33 & 0 \\
\hline UFC e UFPI & 25 & 0 \\
\hline UFC e UFRN & 33 & 33 \\
\hline UFC e UFS & 21 & 0 \\
\hline UFMA e UFPB & 50 & 0 \\
\hline UFMA e UFPE & 83 & 0 \\
\hline UFMA e UFPI & 50 & 0 \\
\hline UFMA e UFRN & 50 & 0 \\
\hline UFMA e UFS & 21 & 0 \\
\hline UFPB e UFPE & 42 & 17 \\
\hline UFPB e UFPI & 43 & 14 \\
\hline UFPB e UFRN & 44 & 22 \\
\hline UFPB e UFS & 30 & 10 \\
\hline UFPE e UFPI & 37 & 0 \\
\hline UFPE e UFRN & 42 & 0 \\
\hline UFPE e UFS & 14 & 14 \\
\hline UFPI e UFRN & 29 & 0 \\
\hline UFPI e UFS & 19 & 0 \\
\hline UFRN e UFS & 15 & 10 \\
\hline Média & 36,58 & 10,33 \\
\hline
\end{tabular}




\section{ENSAIO 9}

Livro 9: Weber, M.; Gerth, H.H. e Mills, C.W. Ensaios de sociologia. 5.ed. Rio de Janeiro, RJ: Livros Técnicos e Científicos, c1982.

Quadro XI. Levantamento dos assuntos catalogados do mesmo livro nos catálogos coletivos online - Livro 9.

\begin{tabular}{|l|l|c|}
\hline Sigla da Biblioteca & Assunto & Classificação \\
\hline UFAL & $\begin{array}{l}\text { Ciências sociais } \\
\text { Sociologia }\end{array}$ & 316.2 \\
\hline UFBA & Ciências Sociais & 316 \\
\hline UFC & $\begin{array}{l}\text { Sociologia - } \\
\text { Discursos, ensaios, } \\
\text { conferências } \\
\text { Classes sociais - } \\
\text { Discursos, ensaios, } \\
\text { conferências } \\
\text { Luta de classes } \\
\text { Burocracia } \\
\text { Carisma }\end{array}$ & 301 \\
\hline UFMA & Sociologia & \\
\hline UFPB & Sociologia & \\
\hline UFPE & Sociologia & 301 \\
\hline UFPI & Sociologia & 301 \\
\hline UFRN & Sociologia & 301 \\
\hline UFS & $\begin{array}{l}\text { Ciências Sociais - } \\
\text { Discursos, ensaios, } \\
\text { conferências } \\
\text { Religião - } \\
\text { Discursos, ensaios, } \\
\text { conferências } \\
\text { Sociologia organizacional } \\
\text { Burocracia }\end{array}$ & 316 \\
\hline
\end{tabular}

Tabela XI. Índices de consistência entre pares de bibliotecas - Livro 9.

\begin{tabular}{|c|c|c|}
\hline $\begin{array}{l}\text { Pares de } \\
\text { Bibliotecas }\end{array}$ & $\begin{array}{l}\text { Índice de } \\
\text { consistência } \\
\text { (relaxado) }\end{array}$ & $\begin{array}{l}\text { Índice de } \\
\text { consistência } \\
\text { (rígido) }\end{array}$ \\
\hline UFAL e UFBA & 50 & 50 \\
\hline UFAL e UFC & 8 & 0 \\
\hline UFAL e UFMA & 50 & 50 \\
\hline UFAL e UFPB & 50 & 50 \\
\hline UFAL e UFPE & 50 & 50 \\
\hline UFAL e UFPI & 50 & 50 \\
\hline UFAL e UFRN & 50 & 50 \\
\hline UFAL e UFS & 20 & 0 \\
\hline UFBA e UFC & 0 & 0 \\
\hline UFBA e UFMA & 0 & 0 \\
\hline UFBA e UFPB & 0 & 0 \\
\hline UFBA e UFPE & 0 & 0 \\
\hline UFBA e UFPI & 0 & 0 \\
\hline UFBA e UFRN & 0 & 0 \\
\hline UFBA e UFS & 12 & 0 \\
\hline UFC e UFMA & 10 & 0 \\
\hline UFC e UFPB & 10 & 0 \\
\hline UFC e UFPE & 10 & 0 \\
\hline UFC e UFPI & 10 & 0 \\
\hline UFC e UFRN & 10 & 0 \\
\hline UFC e UFS & 31 & 12 \\
\hline UFMA e UFPB & 100 & 100 \\
\hline UFMA e UFPE & 100 & 100 \\
\hline UFMA e UFPI & 100 & 100 \\
\hline UFMA e UFRN & 100 & 100 \\
\hline UFMA e UFS & 12 & 0 \\
\hline UFPB e UFPE & 100 & 100 \\
\hline UFPB e UFPI & 100 & 100 \\
\hline UFPB e UFRN & 100 & 100 \\
\hline UFPB e UFS & 12 & 0 \\
\hline UFPE e UFPI & 100 & 100 \\
\hline UFPE e UFRN & 100 & 100 \\
\hline UFPE e UFS & 12 & 0 \\
\hline UFPI e UFRN & 100 & 100 \\
\hline UFPI e UFS & 12 & 0 \\
\hline UFRN e UFS & 12 & 0 \\
\hline Média & 41,14 & 36,44 \\
\hline
\end{tabular}




\section{ENSAIO 10}

Livro 10: Hill, A. A. Aspectos da linguística moderna. 2.ed. São Paulo: Cultrix, 1974.

Quadro XII. Levantamento dos assuntos catalogados do mesmo livro nos catálogos coletivos online - Livro 10.

\begin{tabular}{|l|l|c|}
\hline Sigla da Biblioteca & Assunto & Classificação \\
\hline UFAL & Linguística & 800 \\
\hline UFBA & $\begin{array}{l}\text { Linguística } \\
\text { Linguagem e línguas }\end{array}$ & 801 \\
\hline UFC & $\begin{array}{l}\text { Linguística - } \\
\text { Discursos, ensaios, conferências } \\
\text { Linguagem e línguas - } \\
\text { Discursos, ensaios, conferências }\end{array}$ & 410 \\
\hline UFMA & Linguística & 801 \\
\hline UFPB & $\begin{array}{l}\text { Linguística - } \\
\text { Discursos, ensaios e conferências } \\
\text { Linguagem e línguas - } \\
\text { Discursos, ensaios, conferências }\end{array}$ & 801 \\
\hline UFPE & Linguística & 410 \\
\hline UFPI & Linguística & 410 \\
\hline UFRN & Linguística & 801 \\
\hline UFS & Linguística & 81 '1 \\
\hline
\end{tabular}

Tabela XII. Índices de consistência entre pares de bibliotecas - Livro 10.

\begin{tabular}{|c|c|c|}
\hline $\begin{array}{l}\text { Pares de } \\
\text { Bibliotecas }\end{array}$ & $\begin{array}{l}\text { Índice de } \\
\text { consistência } \\
\text { (relaxado) }\end{array}$ & $\begin{array}{l}\text { Índice de } \\
\text { consistência } \\
\text { (rígido) }\end{array}$ \\
\hline UFAL e UFBA & 50 & 50 \\
\hline UFAL e UFC & 25 & 0 \\
\hline UFAL e UFMA & 100 & 100 \\
\hline UFAL e UFPB & 25 & 0 \\
\hline UFAL e UFPE & 100 & 100 \\
\hline UFAL e UFPI & 100 & 100 \\
\hline UFAL e UFRN & 100 & 100 \\
\hline UFAL e UFS & 100 & 100 \\
\hline UFBA e UFC & 33 & 0 \\
\hline UFBA e UFMA & 25 & 50 \\
\hline UFBA e UFPB & 33 & 0 \\
\hline UFBA e UFPE & 50 & 50 \\
\hline UFBA e UFPI & 50 & 50 \\
\hline UFBA e UFRN & 50 & 50 \\
\hline UFBA e UFS & 50 & 50 \\
\hline UFC e UFMA & 25 & 0 \\
\hline UFC e UFPB & 100 & 100 \\
\hline UFC e UFPE & 25 & 0 \\
\hline UFC e UFPI & 25 & 0 \\
\hline UFC e UFRN & 25 & 0 \\
\hline UFC e UFS & 25 & 0 \\
\hline UFMA e UFPB & 25 & 0 \\
\hline UFMA e UFPE & 100 & 100 \\
\hline UFMA e UFPI & 100 & 100 \\
\hline UFMA e UFRN & 100 & 100 \\
\hline UFMA e UFS & 100 & 100 \\
\hline UFPB e UFPE & 25 & 0 \\
\hline UFPB e UFPI & 25 & 0 \\
\hline UFPB e UFRN & 25 & 0 \\
\hline UFPB e UFS & 25 & 0 \\
\hline UFPE e UFPI & 100 & 100 \\
\hline UFPE e UFRN & 100 & 100 \\
\hline UFPE e UFS & 100 & 100 \\
\hline UFPI e UFRN & 100 & 100 \\
\hline UFPI e UFS & 100 & 100 \\
\hline UFRN e UFS & 100 & 100 \\
\hline Média & 62,25 & 52,78 \\
\hline
\end{tabular}

